\title{
Editorial
}

\author{
Shane Cloude \\ School of Electrical and Electronic Engineering, University of Adelaide, South Australia 5005, Australia \\ Email: scloude@eleceng.adelaide.edu.au
}

\author{
Gianfranco Fornaro \\ Istituto per il Rilevamento Elettromagnetico dell' Ambiente (IREA), Consiglio Nazionale delle Ricerche (CNR), \\ via Diocleziano 32, 80124 Napoli, Italy \\ Email: fornaro.g@irea.cnr.it
}

\section{Fulvio Gini}

Dipartimento di Ingegneria dell'Informazione, Università di Pisa, via Caruso 14, 50126 Pisa, Italy Email:f.gini@iet.unipi.it

\author{
Fabrizio Lombardini \\ Dipartimento di Ingegneria dell'Informazione, Università di Pisa, via Caruso 14, 50126 Pisa, Italy \\ Email:f.lombardini@iet.unipi.it
}

\section{Roland Romeiser}

Institut für Meereskunde, Universität Hamburg, Bundesstraße 53, 20146 Hamburg, Germany

Email: romeiser@ifm.uni-hamburg.de

Synthetic aperture radar (SAR) is a powerful mature technique providing unique high-resolution two-dimensional radio reflectivity images, representing the electrical and geometrical properties of a surface in nearly all weather nightand-day conditions. Range resolution is obtained through signal modulation, while platform motion and synthetic aperture processing allow azimuth resolution. Accurate measurement of radio reflectivity is very useful for vegetation and snow mapping, forestry, land-use monitoring, agriculture, soil moisture determination, mineral exploration, and for oceanography, hydrology, and geophysics.

Interferometric methods, based on further information extraction from the phase difference among at least two complex-valued SAR images, have successfully expanded in the last two decades the remote sensing capabilities of SAR. Depending on the acquisition configuration, SAR interferometry can provide efficient operational topographic mapping or displacement monitoring tools for land and ice applications. Sensitivity to topography with decimeter (from aircraft) to meter (from satellite) accuracy is obtained by the cross-track interferometric configuration, where the SAR acquisitions are separated by a baseline orthogonal to the platform flight path. Cross-track interferometry is finding many applications in remote sensing fields where digital elevation maps (DEMs) are useful, for example, for topographic and urban mapping, geophysics, forestry, hydrology, glaciology, siting for cell phones, and flight simulators. Sensitivity to small surface displacements, of the order of a few millimeters, is obtained by the configuration of differential interferometry, exploiting time acquisition diversity on long (days to years) time scales. Differential interferometry is an established technique for analyzing ground displacements of tectonic nature, for monitoring volcanic areas and slope instabilities, as well as for capturing precursor displacements to building collapses. Another promising SAR interferometry mode allows also ocean surface (or moving vehicle) velocity sensing. This mode is termed along-track interferometry, since it exploits SAR acquisitions separated by a baseline aligned with the platform flight path. It can be regarded as a differential interferometry technique acting on short (fraction of second) time scales to measure decimeter-to-meterper-second velocities. Further details on the various interferometric SAR techniques and their applications can be found in the tutorials papers $[1,2]$.

The need for advanced signal processing techniques within the interferometric SAR processing field is continuously increasing, for improving existing interferometric functionalities, producing novel parameter extraction 
capabilities, and fully exploiting the rich SAR data archives and the potentials originated by new experimented and planned interferometric SAR sensors (multi-baseline airborne and minisatellite cluster systems). Observing this trend, we have put together this special issue. All of the submitted papers went through peer reviews to ensure their correctness, technical significance, and relevance to the special issue. It consists of ten papers on the development of advanced models and new signal processing algorithms in the interferometric SAR field, with an approach mainly oriented towards the exploitation of statistical methods and of baseline/time/frequency/polarization acquisition diversity, to face the challenges of an accurate, reliable, and fully capable interferometric radar remote sensing and to deal with increasingly various and difficult scenarios. In particular, the papers concern the fertilization with, and application of, methods and concepts from the areas of filtering, parameter estimation, spectral estimation, array processing, coherent data fusion, model inversion, detection, and physicalbased statistical modeling. System performance analysis and fuzzy signal processing are also tackled.

The papers are categorized into four interferometric topics: cross-track interferometry, differential interferometry, polarimetric interferometry, and along-track interferometry.

\section{Cross-track interferometry}

Two limiting factors of the standard cross-track interferometry technique are the multiplicative speckle noise, originated by the interaction of the coherent wave radiation with the microscopic structure of the terrain in the resolution cell (distributed scattering), and the $2 \pi$ interferogram ambiguity problems. Fusion of multi-baseline data has been introduced for accurate estimation with reduced noise and phase ambiguity problems. Several works have been carried out in the past on multi-baseline fusion and on the related multifrequency fusion technique.

This area is quite mature, yet recently interest has risen again in multi-baseline/multifrequency fusion through estimators incorporating also spatial (range-azimuth) statistical models. Applications fall mainly in the area of DEM reconstruction, though improvements may be also beneficial for differential interferometry. Also, multifrequency fusion is getting increasing attention thanks to the availability of a growing number of wideband interferometric SAR systems, allowing multifrequency operation through band splitting. In the paper by Budillon et al. multi-baseline/multifrequency cross-track interferometry is formulated in the Bayesian estimation framework, thus leading to the derivation of a maximum a posteriori estimate of the terrain height based on Markov random field a priori statistics.

Modern models of interferometric speckle are derived from optics and are very diffused in the standard interferometry. These are based on a spectral shift theory in the transformed range domain, and can often allow, through special SAR image preprocessing, filtering out the multiplicative noise component from interferometric phase signals, normally occurring on distributed scatterers. The paper by Fornaro et al. extends the classic pixel-based phaseonly multi-baseline fusion towards a slope-based estimation problem. A complex data-level fusion algorithm of multibaseline signals is derived, which allows accounting for the spectral shift effect and thus obtaining an improved multibaseline DEM estimate.

Multi-baseline processing can also be targeted to estimation of multiple signal components, in a framework analogous to that of array processing for direction-of-arrival (DOA) estimation. Array signal processing concepts can be applied to multi-baseline interferometry for separating multiple signals along the elevation direction, allowing a software solution of problems associated with the perspective ambiguities in the so-called layover geometries. In its more general form, multi-baseline processing can be cast in an imaging framework of continuous radar reflectivity distributions along the elevation direction. The resulting $3 \mathrm{D}$ SAR tomography technique allows full three-dimensional imaging of semitransparent volume scattering layers, for example, for forest structure investigation. Multi-baseline separation of geometrical superposition effects is an emerging area where recent work has resulted in significant advances. In the paper by Lombardini and Gini the problem of estimating the number of multiple distributed scatterers is investigated. Model order selection (MOS) is crucial both for correctly applying superresolution algorithms and for interpreting the results of nonparametric-Fourier-based or adaptive-algorithms. MOS becomes a nonconventional problem in the presence of speckle, which is the case for the typical scenario of extended layover terrain patches analyzed in the paper. Sensitivity to speckle of classical eigenvalues-based MOS methods and system tradeoffs are numerically investigated. MOS algorithms robustification through diagonal loading is also considered.

When multiple baselines or multiple acquisition frequencies are not employed, as it is still frequent for airborne DEM production, solving the classical interferogram ambiguity to derive absolute phase measurements is an extremely important step in the interferometric processing chain. Interferometric phase unwrapping has become a quite mature subject after concepts from network flow programming were introduced. However, performance improvement may be still possible by refining spatial filtering which is important to enforce a smoothness constraint of the phase field, on which unwrapping relies. Also, room for research exists to incorporate the temporal dimension in the spatial unwrapping procedure in the case of repeat-pass multi-baseline acquisition for differential interferometry.

The next paper by Aiazzi et al. addresses the former problem by an original application of fuzzy logic to restoration of phase images, which are typically corrupted by nonstationary noise. Space-varying filtering is stated as a blind structural estimation problem of matching pursuit, in which multiple estimators are calculated and used in a finite series expansion in a fuzzy fashion. Simulated and real-data results show that the concept is promising. 


\section{Differential interferometry}

This interferometric mode has been investigated for a long time and is now in an operational status, yet there is still room for improvements. Being a method intrinsically based on repeat-pass acquisition, limitations of the basic differential interferometric technique are due to possible atmospheric propagation phase artefacts and long-term temporal decorrelation of the surface scattering. The possible baseline speckle decorrelation is an additional limiting factor. These problems are overcome by investigation of long-time series and proper processing of data from highly coherent scatterers. Well-known permanent or persistent scatterers (PS) techniques have been already successfully applied to monitoring of ground deformation in many areas around the world.

However, interference among multiple signal components within a ground resolution cell, occurring especially in urban areas, may lead to a reduction of the number of monitored pixels and thus of the coverage density. The paper by Ferretti et al. addresses this problem and discusses an improvement of the standard PS technique based on the adoption of parametric modeling, thus leading to the concept of higher-order differential interferometry. It is shown how the PS density can be increased at the price of a higher computational load.

Differential interferometry can also be used for coherent detection of small surface changes. Traditionally, coherent change detection has been based on empirical observations of changes with little or no physical understanding of the source of these changes. The paper by Williams and Preiss demonstrates application of the latest coherent electromagnetic scattering modeling techniques to an analysis and clearer understanding of the source of signal changes in SAR interferometry. Such techniques provide the basis for a more physics-based approach to the understanding and analysis of some temporal decorrelation effects in radar interferometry.

\section{Polarimetric interferometry}

Multi-baseline processing allows distinguishing multiple superimposed scatterers in the elevation direction. An alternative or additional separation capability for parameter estimation of multiple-layered volumetric scatterers has been obtained by crossing polarimetric SAR with SAR cross-track interferometry. The resulting polarimetric SAR interferometry technique is based on inversion of multichannel (multipolarisation or multipolarisation and multi-baseline) scattering models to coherently separate the contributions of different scattering mechanisms. This approach leads to the possibility of extracting important vegetation structure parameters such as forest height and biomass even from a single-frequency single-baseline interferometer. In its multi-baseline form, it offers the potential for improved and refined 3D structure estimation in vegetation mapping as well as in land ice and snow thickness monitoring.

One of the key problems in polarimetric interferometry is devising a rigorous statistical model which accounts not only for the classical multiplicative speckle noise but also for an additive noise component due to coherence estimation. The paper by López-Martínez et al. describes a new model intended to highlight the relative contributions of these two components and hence to help in the estimation and filtering of polarimetric interferometric data.

The next paper by Krieger et al. concerns a new method for analyzing system performance in polarimetric interferometry. They introduce the concept of a phase tube which is a multipolarisation extension of the more classical single-channel coherence analysis. This tube concept is demonstrated by application to various future space-based radar scenarios. The concept provides an important unifying framework for the design of future multipolarisation interferometers.

\section{Along-track interferometry}

The acquisition configuration of this interferometric mode is analogous to that of the displaced phase center antennas (DPCA) and space-time adaptive processing (STAP) techniques. Despite the large potentials for high-resolution radar remote sensing of ocean surface currents, waves, and other dynamical features, along-track interferometry is still much less developed and less popular than cross-track and differential interferometry. This results partly from the fact that the retrieval of surface current fields or wave spectra from interferometric radar images is somewhat difficult and requires complex inversion techniques. This limitation is not yet fully overcome. Along-track interferometry can also be employed for land applications such as traffic monitoring. However, the possible short decorrelation times of the backscattered signal from the ocean and other moving targets on the order of milliseconds, as well as the relatively high velocities of interest, may require an interferometric configuration with two antennas on a single platform, which has not been available on (civilian) satellites until now. Finally, the variety of applications and the commercial potential of along-track interferometry seem to be relatively limited. However, first satellites with along-track interferometry capabilities will be launched in the near future, and latest developments indicate a particularly vital interest in the technology for moving target indication and traffic monitoring activities. Also these important developments are covered by two papers in this issue.

The paper by Chiu deals with moving target indication via along-track interferometry and the use of a novel fractional Fourier transform technique which leads to a significant improvement of the signal-to-noise ratio in velocity and position estimates for moving targets. The proposed method is compared with a more conventional approach and applied to airborne interferometric SAR data for a demonstration.

The last paper by Moccia and Fasano discusses the potential of complementing the upcoming Italian COSMOSkyMed mission, which consists of four small satellites with SAR sensors with the main objective to monitor the Mediterranean area, by a fifth satellite for (cross-track and along-track) interferometric measurements. Various possible orbit constellations are presented and evaluated in 
terms of measuring capabilities and availability of desired interferometric baseline configurations in different geographical regions.

Also, in the paper by Budillon et al. mentioned in the cross-track interferometry section, the multi-baseline/multifrequency concepts are extended to the along-track processing. Interferometric phase statistics in presence of clutter are computed and a maximum likelihood velocity estimation algorithm for moving targets, which exploits only phase signals acquired by different channels, is derived.

Finally, we would like to thank all the authors who responded to our Call for Papers for their contributions. We would also like to express our thanks to all the reviewers for dedicating their precious time to provide detailed reviews. We would also like to acknowledge the support of Professor Marc Moonen from EURASIP JASP's Editorial Board. With the help of all these contributors, we have fulfilled our expectations, and hope that this special issue offers a considerable and timely contribution to the area of signal processing for interferometric synthetic aperture radar.

\section{Shane Cloude Gianfranco Fornaro Fulvio Gini \\ Fabrizio Lombardini Roland Romeiser}

\section{REFERENCES}

[1] R. Bamler and P. Hartl, "Synthetic aperture radar interferometry," Inverse Problems, vol. 14, pp. R1-R54, 1998.

[2] P. A. Rosen, S. Hensley, I. R. Joughin et al., "Synthetic aperture radar interferometry," Proc. of the IEEE, vol. 88, no. 3, pp. 333 382,2000 .

Shane Cloude was born in Chatham, England, in 1959. He obtained his B.S. degree (with honors) from the University of Dundee, Scotland (1981), and his Ph.D. degree from the University of Birmingham, England (1987), both in electrical and electronic engineering. He worked at the Royal Signals and Radar Establishment (RSRE) in Malvern, England, before taking up teaching and research positions at the Universi-

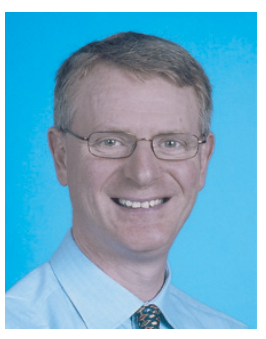
ties of Dundee in Scotland, York in England, and Nantes in France. In 1996 he was the recipient of a fellowship from the Alexander von Humboldt Foundation in Germany, working in collaboration with DLR on the development of polarimetric SAR interferometry. Between 1997 and 2004, he was the Director of Applied Electromagnetics (AELc), a research consultancy company based in Scotland, undertaking contract research in radar and antenna design for industry, government, and defence laboratories. In 2004 he was appointed to the DSTO Chair in Microwave Radar at the University of Adelaide in Australia. His main research interests are in the exploitation of polarization effects in radar and optical remote sensing. Shane Cloude is a Fellow of the IEEE (2001) and an Associate Editor of the IEEE Transactions on Geoscience and Remote Sensing.
Gianfranco Fornaro received the Laurea degree in electronic engineering from the University of Napoli in 1992 and the Ph.D. degree from the University of Roma "La Sapienza" in 1997. He is a Senior Researcher at the Istituto per il Rilevamento Elettromagnetico dell'Ambiente (IREA) of the Italian National Research Council (CNR); he has been also an Adjunct Professor of telecommunications at the Universities of

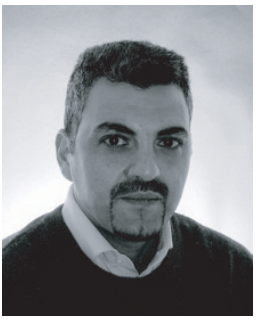
Cassino and Napoli Federico II, and of signal theory at the University of Reggio Calabria. His main research interests regard the signal processing field with applications to synthetic aperture radar (SAR) data processing, SAR interferometry, differential SAR interferometry for airborne and spaceborne systems, and multichannel processing for coherent SAR image fusion. Dr Fornaro has been a Visiting Scientist at the German Aerospace Establishment (DLR) and at the Politecnico of Milano; he has been also an Invited Lecturer at the Istituto Tecnologico de Aeronautica (ITA) in Sao José dos Campos (Brazil) and at RESTEC (Tokyo). Dr. Fornaro has been invited as the Chairman and Cochairman at several international conferences; he was a member of the technical program committee for the TIWRS 2003. He is also responsible for the Remote Sensing Unit of the Regional Center of Competence "Analysis and Monitoring of the Environmental Risk" supported by The European Community on Provision 3.16. He has authored more than thirty international peer-reviewed journal papers and almost sixty papers in proceedings of international conferences in the SAR processing field. Dr. Fornaro was awarded in 1997 the Mountbatten Premium by the Institution of Electrical Engineers (IEE).

Fulvio Gini received the Doctor Engineer (cum laude) and the Research Doctor degrees in electronic engineering from the University of Pisa, Italy, in 1990 and 1995, respectively. In 1993 he joined the Department of Ingegneria dell'Informazione of the University of Pisa, where he has been an Associate Professor since October 2000. From July 1996 to January 1997, he was a visiting researcher at the Department of Electrical

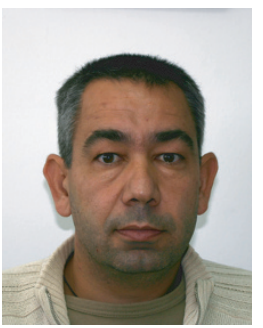
Engineering, University of Virginia, Charlottesville. He is an Associate Editor for the IEEE Transactions on Signal Processing and a Member of the EURASIP JASP Editorial Board. He was the corecipient of the 2001 IEEE AES Society's Barry Carlton Award for Best Paper. He was the recipient of the 2003 IEE Achievement Award for outstanding contribution in signal processing and of the 2003 IEEE AES Society Nathanson Award to the Young Engineer of the Year. He is a Member of the Signal Processing Theory and Methods (SPTM) Technical Committee (TC) of the IEEE Signal Processing Society and a Member of the Sensor Array and Multichannel (SAM) TC. He is a Member of the Administrative Committee of the EURASIP Society and the Awards Chairman. He is the Technical Chairman of the 2006 EURASIP Signal and Image Processing Conference (EUSIPCO), Florence, Italy, September 2006. His research interests include modeling and statistical analysis of radar clutter data, non-Gaussian signal detection and estimation, parameter estimation, and data extraction from multichannel interferometric SAR data. He authored or coauthored more than 70 journal papers and 70 conference papers. 
Fabrizio Lombardini received the Italian Laurea degree (with honors) in electronic engineering and the Ph.D. degree in telecommunication engineering from the University of Pisa, Italy, in 1993 and 1997, respectively. He was then granted by the EU a Marie Curie Fellowship of the Training and Mobility of Researchers (TMR) Program. He spent the program period as a postdoctoral researcher at the Department

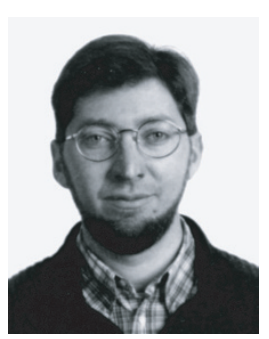
of Electronic and Electrical Engineering of University College London, UK, from 1998 to 1999. Then, he joined the Department of "Ingegneria dell'Informazione" of University of Pisa, where he currently holds the position of Assistant Professor. He is an IEEE Member since 1993 and a Senior Member since January 2003. He has given lectures at universities and institutions in Italy and abroad, and has chaired special sessions at international conferences. He is the coauthor of a tutorial entitled "Multi-baseline post-processing for SAR interferometry" presented at the IEEE Sensor Array and Multichannel Workshop (July 2004). His general interests are in the areas of statistical signal processing, estimation and detection theory, adaptive and superresolution spectral analysis, array processing, and performance bounds evaluation, with application to radar systems. In particular, his research interests include multi-baseline and multifrequency interferometric SAR algorithms and systems, both cross- and along-track, three-dimensional SAR tomography, differential SAR interferometry, multisensor data fusion, and radar detection in non-Gaussian clutter.

Roland Romeiser received the Diploma degree in physics from the University of Bremen, Germany, in 1990 and the Dr.rer.nat. degree from the University of Hamburg in 1993. He is now a permanent Staff Scientist at the Institute of Oceanography of the University of Hamburg. He has wide experience in the remote sensing of ocean currents, waves, and winds by various mi-

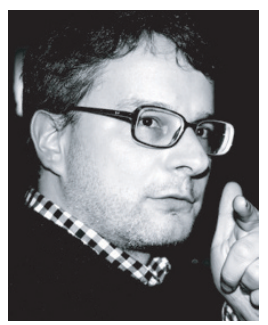
crowave sensors. He has been involved in a number of national and international remote sensing projects and experiments, and he has organized and chaired several sessions at international remote sensing conferences. His current research focuses on the theoretical modeling of SAR and InSAR signatures of spatially varying ocean surface currents and the development and evaluation of current retrieval techniques. From August 1998 through July 1999, Dr. Romeiser spent a year at the Johns Hopkins University / Applied Physics Laboratory as a Feodor Lynen Fellow of the Alexander von Humboldt Foundation. In fall 2000 he became an Associate Editor of the IEEE Journal of Oceanic Engineering for topics dealing with radar imaging and interferometry. 